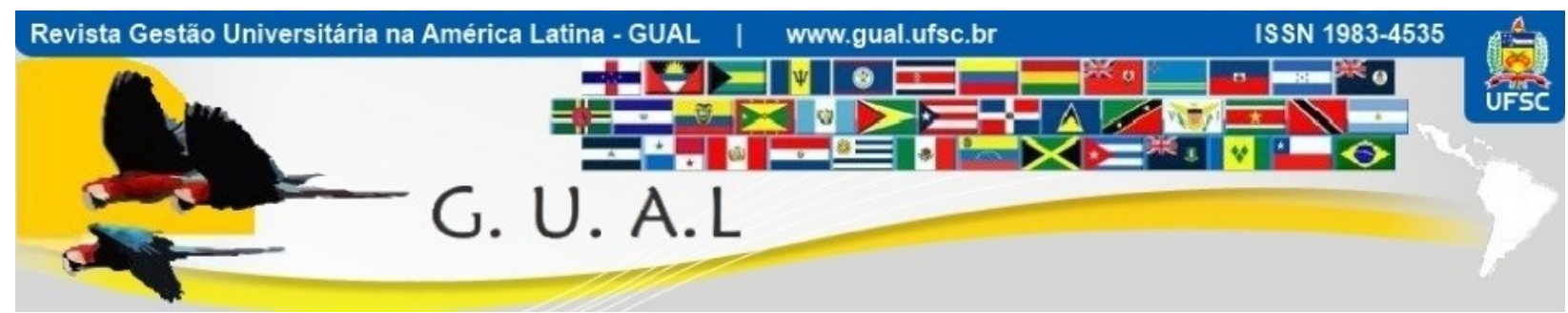

DOI: http://dx.doi.org/10.5007/1983-4535.2015v8n3p261

\title{
GUARDA E MANIPULAÇÃO DE DOCUMENTOS ELETRÔNICOS E SEUS EFEITOS NA CULTURA ORGANIZACIONAL EM UMA UNIVERSIDADE
}

\section{STORAGE AND HANDLING OF ELECTRONIC DOCUMENTS AND ITS EFFECTS ON ORGANIZATIONAL CULTURE AT UNIVERSITY}

Leonardo Roth, Mestre

Universidade de Caxias do Sul - UCS

$\underline{\text { lroth@ucs.br }}$

Roberto Birch Gonçalves, Doutor Universidade de Caxias do Sul - UCS rbgoncal@ucs.br

Marco Aurélio Bertolazzi, Doutor Universidade de Caxias do Sul - UCS mabertol@ucs.br

Ana Cristina Fachinelli, Doutora Universidade de Caxias do Sul - UCS afachinelli@gmail.comrofe

Recebido em 18/dezembro/2013

Aprovado em 15/julho/2015

Sistema de Avaliação: Double Blind Review

Esta obra está sob uma Licença Creative Commons Atribuição-Uso. 


\title{
RESUMO
}

Os documentos digitais se tornam cada vez mais presentes na rotina das Instituições de Ensino Superior (IES) ampliando a preocupação com a integridade dos dados, a garantia da identidade e a confidencialidade das partes envolvidas. A legislação obriga a IES a manter a guarda dos documentos dos alunos, avaliações, registros de notas e assiduidade por anos e até por guarda perpétua. Portanto, quando uma IES adota um modelo para a guarda, tratamento e validação dos documentos em meio eletrônico ou digital adota, também, novos processos de gestão da informação que podem afetar a cultura organizacional. O objetivo deste estudo foi identificar e analisar as interfaces entre a inovação na gestão eletrônica de documentos e a cultura organizacional relacionado a mudanças de processo na guarda eletrônica de documento realizando-se um estudo de caso numa IES comunitária. Os resultados indicam que, embora existam dificuldades de adaptações ao processo devido à cultura de uma IES, com o amadurecimento do processo o aprendizado amplia os benefícios do novo processo pela disseminação da inovação e seus reflexos em outras áreas. $\mathrm{O}$ estudo concluiu que o processo de digitalização além de trazer vantagens onde foi aplicado, estendeu os benefícios para outras áreas da IES.

Palavras-chave: Gestão Eletrônica de Documentos. Universidade. Cultura organizacional.

\begin{abstract}
Digital documents become increasingly present in the routine of people and Higher Education Institutions (HEI) increasing the concern with the integrity of data, assurance of identity and confidentiality of the parties involved. The universities must, by law, keep the custody of student's documents, records of grades and absences, ratings and so on, for years even for perpetual guard. Therefore, when a HEI adopt a model of custody, treatment and validation of documents in electronic or digital media, also adopts new management processes of information that may affect the organizational culture. The objective of this study was identify $\mathrm{e}$ analyze the interfaces between innovation in electronic document management and organizational culture related to process changes in electronic document custody through a case study at a community HEI. The results shows that, though there are adaptation difficulties to the process because of the HEI culture, with the maturation of the process, learning expands the benefits of the new process through dissemination of innovation and their reflects in other areas. The study concludes that the scanning process also brings advantages where it was applied, the benefits extended to other areas of the HEI.
\end{abstract}

Keywords: Electronic Document Management. University. Organizational Culture. 


\section{INTRODUÇÃO}

A vivência em um universo digital tem crescido atingindo praticamente todos os aspectos da sociedade. Atividades como pagamentos e depósitos bancários, músicas digitais, ecommerce, comunicação telefônica e-mails e outros meios fazem parte da rotina de sociedades com bons níveis de desenvolvimento em termos de tecnologias de informação e de comunicação. Fox e Rowe (1999) abordam que se está construindo um mundo digital que inclui o ciberespaço, infraestrutura de informação global, era da informação, (super) rodovias da informação, interespaço e uma sociedade sem papel.

Marques (2005) destaca que, pela evolução social, política, econômica e jurídica, a definição dos documentos eletrônicos é necessária tendo em vista que "o documento tradicional não mais se adapta à necessidade moderna em propiciar uma maior agilidade à circulação da informação" (p. 125). Assim, o poder do aperfeiçoamento digital, as possibilidades para organização das mídias e a matemática de compressão e comunicação alteram o conceito de geração, preservação e otimização onde, o acesso ao conhecimento registrado, deve ser de maneira pronta, oportuna e com informação relevante (WATERS, 2001; CONWAY, 2001).

Nesse contexto, as organizações articulam políticas de segurança que são procedimentos e medidas técnicas servindo como ferramentas para coibirem o acesso indevido ou não autorizado, além de impedir a realização de alteração, roubo ou danos físicos ao sistema ou documentos (LAUDON e LAUDON, 2004).

Muitos autores (Porter, 1980; Davenport e Prusak, 1998) destacam que as informações rápidas e confiáveis, precisas e oportunas podem auxiliar as organizações em sua sobrevivência, e crescimento sustentável nos ambientes em constante mutação. Além disso, a aprendizagem organizacional e a gestão do conhecimento passam a serem instrumentos eficazes para vencer os desafios relacionados ao seu contexto ambiental (AUSTER e CHOO, 1995; NONAKA e TAKEUSHI, 1997; SENGE, 1999).

O crescente fluxo informações tornou-se um dos principais elementos de mudanças das organizações a partir de dados da internet e outras fontes eletrônicas onde o gerenciamento, dessas informações deve fomentar novas fontes de valor econômico, proporcionado novos insights para a ciência - The Economist (2010).

Nesse cenário globalizado, os dados individuais ou das organizações não permanecem mantidos e protegidos em registros manuscritos ou impressos, separados pelas unidades de 
negócios ou pelos interesses da pessoa onde o sistema virtual, passa a concentrar e arquivar os dados de computador podendo ser acessado "facilmente por grande número de pessoas e por grupos externos à organização. Consequentemente os dados automatizados são mais suscetíveis à destruição, fraude, erro e uso indevido" (LAUDON e LAUDON, 2004, p. 461).

Devido à facilidade de alterar, duplicar, plagiar e manipular os documentos digitais e virtuais sem deixar vestígios, cada vez mais aumenta a preocupação com a veracidade das informações e dos documentos, ao contrário dos papéis que são de difícil falsificação e se tem inúmeros meios para que se possam evitar tais ações (WILSON, 1999).

No caso das instituições de ensino esta situação é particularmente importante, pois um sistema que não garanta segurança para um diploma ou certificação acadêmica pode colocar disponível para a população um profissional inabilitado. Além disso, uma IES se fundamenta na sua tradição e história e, seus documentos, na maioria possuem guarda perpétua.

Assim, o intenso crescimento na geração e a necessidade de responsividade na recuperação de documentos nas IES podem evidenciar dificuldades nos processos internos geradores de custos e dificuldades cotidianas. Portanto, instrumentalizar a organização com ferramentas que proporcionem a captura (ou conversão) de documentos físicos, gerenciando seus fluxos de armazenagem e recuperação é fator fundamental para redução de custos, erros e eficiência interna da IES. Para cumprir esta função pode-se utilizar o GED.

O objetivo principal deste trabalho foi o de identificar e analisar as inovadoras interfaces da gestão eletrônica de documentos e sua relação com a cultura organizacional decorrente das mudanças de processo. Para tanto foi realizado um estudo de caso numa IES comunitária do Estado do Rio Grande do Sul.

Como resultado geral obteve-se a certeza da melhoria dos processos internos, tanto em termos econômicos como de precisão, mas o custo social de adaptação foi igualmente intenso.

\section{REFERENCIAL TEÓRICO}

Este referencial aborda as questões relacionadas às vantagens produzidas pela modernização da guarda de documentos e da certificação digital, pois são elementos importantes sob o ponto de vista da gestão cotidiana das IES. Na sequencia buscou-se apurar os reflexos destas ações a luz da cultura organizacional específica das IES. 


\subsection{GUARDA DIGITAL DE DOCUMENTOS}

O GED ou gerenciador eletrônico de documentos é um sistema de indicação e localização de documentos, dando acesso a uma variedade de containers de informação que podem conter imagens, sons e documentos eletrônicos (RHEINNER GROUP, 2000). Para Koch (1997) o GED vai mais além e faz o gerenciamento de todo o ciclo de vida das informações desde sua criação até o seu arquivamento. Este ciclo envolve a cadeia de valor em torno do processo de transformação de dados em informações e estas em conhecimento. No âmbito das organizações esse processo está imbricado com a própria vida da organização já que há atributos informacionais acoplados à diferentes atividades desenvolvidas por distintos setores. Assim quando ocorre uma mudança no modelo de gestão de documentos tradicional para o GED há implicações sobre as práticas organizacionais que por sua vez podem afetar a própria cultura da organização.

Assim, o GED ao fim e ao cabo se caracteriza por ser um processo inovador para as IES, pois no momento que a esta promove o desenvolvimento de seus processos e método de forma tão radical quanto o GED, incorporará qualidade em seus produtos e serviços estimulando o crescimento do negócio ao longo do tempo, havendo forte relação entre inovação e o sucesso organizacional se refletindo em "efetividade", "lucratividade" e a “satisfação do consumidor" (HART, 1996; CUMMING, 1998; JOHNE, 1999).

Além disso, recentemente verifica-se o crescimento do dinamismo e das mudanças organizacionais e da inovação onde as estratégias organizacionais também se tornaram dinâmicas e mais flexíveis em prol da competitividade de mercado. Inovação requer que as organizações sejam redesenhadas, ou seja, novas formas de gestão, de organização, de estratégias, de orientação e de transformação devem indicar o caminho para a mudança contínua e inovação como sinônimo de crescimento (CHANAL, 2004).

Muitas soluções em GED - Gerenciamento Eletrônico de Documentos concentram-se em digitalizar os documentos mantendo os arquivos em prédio ou local separado, muitas vezes de maneira inadequada. Essa solução faz com que o investimento traga apenas a solução para a busca e manuseio rápido do documento, sem a validade jurídica necessária para o descarte tendo que, muitas vezes, manter grandes equipes para o gerenciamento dos dois arquivos (físico e digital).

Outra solução constantemente encontrada é a digitalização dos documentos e a posterior microfilmagem ou, a partir da microfilmagem, torná-los documentos eletrônicos 
que, por sua vez, possuem as mesmas fragilidades elencadas anteriormente onde o gerenciamento deve ser duplicado, pois se trata de dois modelos de arquivamento de documentos.

Ressalta-se também que as temporalidades de documentos apesar de haver padronização de procedimentos pelo Conselho Nacional de Arquivos - CONARQ a temporalidade, que é o instrumento que indica o tempo e a permanência dos documentos em arquivos correntes e intermediários e sua destinação final é apenas um indicativo de padronização, visto que cabe a cada órgão, considerando sua especificidade e legislação estabelecer os seus prazos e consequentemente sua tabela temporal (CONARQ, 2012).

Já a PORTARIA No 255, de 20 de dezembro de 1990, da Secretaria Nacional de Educação Superior - Ministério da Educação - SeSu, estabelece, em seu Art. 7. ${ }^{\circ}$, que o arquivamento de documentos poderá ser realizado com a adoção de: i) Encadernação da ficha original correspondente a cada ano de atividade encerrada; ii) Microfilmagem; iii) Sistema computadorizado.

A partir da promulgação da Lei $\mathrm{n}^{\circ} 5.433$, de 8 de maio de 1968 que regula a microfilmagem de documentos oficiais e do Decreto $\mathrm{n}^{\circ} 1799$ de 30 de janeiro de 1996 que Regulamenta a Lei $\mathrm{n}^{\circ}$ 5.433, muitas instituições e empresas, a partir dessa tecnologia, avançada para o seu tempo, passam a criar políticas de tratamento e descarte de documentos. Esse sistema, que a partir de microfotografias dos documentos em filme especial com posterior revelação e emissão de cópia de segurança propiciam o descarte dos documentos ou, a critério da empresa, a permanência do documento físico em outro local como "arquivo morto", desnecessário, mas comum pelo desconhecimento real da legislação (BRASIL, 2012).

Tal tecnologia está presente ainda hoje em muitas organizações pública ou privada, como solução para o destino final de documentos. Observa-se também a conversão dos filmes em arquivo digital, buscando assim agilizar o resgate dos documentos, o que não parece ser uma solução ruim.

Muitas empresas também terceirizam a microfilmagem, recebendo apenas os filmes ou mídias (CD ou DVD) com as fotos e relatórios com a indexação dos documentos e, ainda assim a terceirização dos arquivos para empresas especializadas em guarda dos documentos.

Nesse sentido, Conway (2001) destaca que, gerar cópias de preservação em microfilme sem tornar possível sua localização ou a dificuldade e restrição do manuseio está 
em desencontro com as políticas de arquivos em países democráticos e não podem ser apenas um simples modelo de armazenagem de documentos.

No Brasil, a Portaria $n^{\circ} 1.224$ de 18 de dezembro de 2013 especifica que os acervos acadêmicos das IES pertencentes ao sistema federal de ensino obedeçam às normas constantes no Código de Classificação de Documentos de arquivos constantes em tabela de temporalidade especificadas em sistema de gestão de documentos próprios da administração pública federal (BRASIL, 2013f). Esse sistema, instituído pela Lei 4.915 de 12 de dezembro de 2003, conforme o Art. 2 "garantir ao cidadão e aos órgãos e entidades da administração pública federal, de forma ágil e segura, o acesso aos documentos de arquivo e às informações neles contidas, resguardados os aspectos de sigilo e as restrições administrativas ou legais" (BRASIL, 2013e).

Destarte, considerando que o "sistema computadorizado" mencionado na Portaria $\mathrm{SeSu}^{\circ} 255$, não especifica as metodologias de guarda e disponibilização dos dados permite que as instituições mantenham parte de seus acervos virtualizados, não excluindo a obrigatoriedade de guardas físicas que, pela fé pública das instituições de ensino, validam a guarda virtualizada desde que obedecido aos padrões de conversão fiel de um documento físico para digital com a aplicação de certificado ICP-Brasil (BRASIL, 2012).

Em termos de tendência é possível visualizar a evolução das tecnologias no sentido de tratarem altos volumes de dados. Big Data é uma técnica que trata os dados considerando a lógica dos 5Vs (velocidade, volume, variedade, veracidade e valor) em termos de informações disponibilizadas (Big Data, 2011). Estas informações, por sua vez estão estruturadas, com bom nível de organização, disponibilizadas por governos, entidades nacionais e internacionais, organizações setoriais, reforçando a necessidade de avançarem-se os estudos e as publicações sobre o gerenciamento de dados e documentos eletrônicos e a respectiva certificação digital considerando-se este novo paradigma.

\subsection{CERTIFICAÇÃO DIGITAL}

Desde 1996 quando a Organização das Nações Unidas (ONU), junto a Comissão das Nações Unidas para o Direito Comercial Internacional (UNCINTRAL) transformou-se na provedora da certificação mundial. A regulamentação da Certificação Digital trouxe a padronização mínima a ser seguida pelos membros onde hoje todos os Países signatários compõem a rede de acreditação (UNCITRAL, 2012). 
A certificação digital foi desenvolvida para assegurar a não manipulação dos documentos digitais, desde que, assinados e certificados digitalmente. Tal processo garante a integridade, a confidencialidade, a validade jurídica a autenticidade, e o não repúdio dos documentos, pois estão protegidos contra qualquer tipo de alteração, acesso e uso não autorizado (PEREIRA, 2012).

De acordo com Wilson (1999) a segurança tem cinco aspectos importantes que fazem com que um documento digital tenha força e veracidade, Quadro 1.

\begin{tabular}{|c|c|c|}
\hline Aspectos & Função & Exemplos \\
\hline Autenticidade & $\begin{array}{l}\text { Identifica se o } \quad \text { documento } \\
\text { verdadeiro ou não }\end{array}$ & $\begin{array}{l}\text { Folha timbrada; registros específicos } \\
\text { das organizações }\end{array}$ \\
\hline Integridade & $\begin{array}{l}\text { Dificuldade de mudança no papel } \\
\text { ou documento }\end{array}$ & $\begin{array}{l}\text { Marcas em relevo ou de difícil } \\
\text { reprodução }\end{array}$ \\
\hline Não repúdio & Segurança da assinatura & Reconhecimento de firma notarial \\
\hline Confidencialidade & \begin{tabular}{|llll}
$\begin{array}{l}\text { Garantia } \\
\text { exclusiva }\end{array}$ & leitura única \\
\end{tabular} & $\begin{array}{l}\text { Conferencia de entrega contra } \\
\text { documento identificador }\end{array}$ \\
\hline Disponibilidade & Certeza e facilidade da entrega & Sistema de correios \\
\hline
\end{tabular}

Quadro 1 Aspectos que promovem a veracidade de um documento digital Fonte: Baseado em Wilson (1999)

Para Hassler e Biely (1999) a autenticidade garante que uma entidade é realmente quem ela é, autenticação e integridade de dados que assegura as origens dos dados e a não manipulação dos mesmos e o não-repúdio, ou seja, que não pode ser negado, ex.: a criação, envio e recebimento da informação.

Já para Marques (2005, p. 167) a assinatura digital "é um método que garante que determinada mensagem não seja alterada durante seu trajeto". Para a Certsign (2014) certificado digital é a identidade no meio eletrônico que permite, por sua validade jurídica, realizar diversos serviços na esfera digital, com facilidade de acesso e redução de custos.

Para dar validade jurídica aos documentos estes devem estar certificados por Autoridade Certificadora (AC), vinculada a Infraestrutura de Chaves Públicas brasileira (ICP - BRASIL), compreendendo esta a cadeia hierárquica e de confiança que viabiliza a emissão de certificados digitais para identificação do cidadão quando efetuado no meio virtual (BRASIL, 2012a).

No Brasil, o Instituto Nacional de Tecnologia da Informação (ITI) é o órgão federal vinculado à Presidência da República, que tem o objetivo de manter a Infraestrutura de Chaves Públicas Brasileiras - ICP Brasil, sendo a primeira autoridade da cadeia de certificação - AC Raiz (BRASIL, 2012). O certificado ICP-Brasil tem sua aceitabilidade 
garantida pela Medida Provisória n. ${ }^{\circ}$ 2.200-2, datada de 24 de agosto de 2001, que institui a Infraestrutura de Chaves Públicas Brasileiras ICP-Brasil e dá outras providências onde (BRASIL, 2012f):

“Art. 10. Consideram-se documentos públicos ou particulares, para todos os fins legais, os documentos eletrônicos de que trata esta Medida Provisória.

$\S 1^{\mathrm{o}}$ “As declarações constantes dos documentos em forma eletrônica produzidos com a utilização de processo de certificação disponibilizado pela ICP-Brasil presumem-se verdadeiros em relação aos signatários, na forma do art. 219 do Código Civil””.

O Ministério da Educação (MEC), através da Portaria Ministerial n. ${ }^{\circ}$ 40, datada de 12 de dezembro de 2007, inovou seus processos ao exigir das instituições que o trânsito de documentos passe a ser meio eletrônico e em sistema específico. Assim os processos regulatórios dos cursos de educação superior, bem como notificações e atos, transitam eletronicamente desde que de acordo com os requisitos do ICP-Brasil (MEC, 2012).

Com isso, a preocupação da segurança aumentou e, inicialmente acentuou-se a partir das transações bancárias pela Internet que tem necessitado urgentemente de soluções que resolvam o problema de insegurança e também de dados não fidedignos, surgindo assim às assinaturas eletrônicas. Lincoln (2004) acredita que a ferramenta de autenticação é um dos mais seguros meios de assinaturas tecnológicas no mercado. Smedinghoff e Bro (1999) descrevem a assinatura digital como um tipo de tecnologia específica de um método de assinatura eletrônica, que é criado através da utilização de processos de criptografia de chave pública e privada.

A representação da assinatura eletrônica ou digital se dá através dos dados que identificam o autor do documento, sendo normalmente criada para encriptar o conteúdo através de um algoritmo criptografado, ou seja, através de uma combinação numérica gerada pelo autor do documento, fazendo com que somente o autor ou quem possuir esta chave de acesso possa de fato ter acesso aos dados informados. Para que uma ou várias pessoas possam acessar tal documento, são distribuídas chaves de acesso onde a identificação se dá pelo certificado digital (WILSON, 1999).

Para que as chaves emitidas não sejam alteradas ou manipuladas no trânsito da informação, se criou os certificados digitais, que trabalham de forma padronizada, uniforme e sistemática para a distribuição das mesmas, garantindo a autenticidade e integridade das informações (WINN, 2001; BURNETT; PAINE, 2002; FREEMAN, 2004). 
Os certificados digitais são emitidos por autoridades certificadores que assumem a responsabilidade de identificar os usuários combinando-os com as chaves de acesso. Cada certificado é um documento eletrônico e contém uma cópia da chave de acesso, cada chave de acesso possui um controle de acesso (CA) que atesta a propriedade particular de cada chave de acesso. Estas chaves são principalmente utilizadas para autenticação de acessos via Internet, seja ele em compras pela internet ou acesso a documentos.

Portanto, o certificado digital assemelha-se com carteira de identidade ou passaporte onde identifica a pessoa física ou jurídica com informações importantes sobre seu titular, o que também garante a autenticidade (GAVILANES, 2005).

Diante do exposto pode-se depreender que o processo de eletronização com certificação digital de documentos promove, numa IES, dificuldades de adaptação e ajustes que são naturais pela novidade, mas que são agravados pela tradição de serviços manualizados.

\subsection{CULTURA ORGANIZACIONAL}

Apesar de a cultura organizacional ser um tema que surgiu durante a década de 1960, é a partir dos anos 1980 que começa a ser travado um intenso debate a respeito da sua natureza e importância para o campo administrativo. O primeiro autor que utilizou tal expressão com um sentido próximo ao conceito atual foi Pettigrew (1979). Smircich (1983) faz uma classificação oportuna, dividindo os estudos da área entre os que consideram a cultura organizacional como uma variável e os que a tratam como uma metáfora para a própria organização.

A evolução do campo é marcada por aquilo que Martin e Frost (2001) chamam de jogos de guerra. Assim, Martin (2002) relata o surgimento de grupos divergentes sobre o tema, o que implica em produção de conhecimento não cumulativo na área. A primeira e mais conhecida perspectiva é a da integração, formada por pesquisadores que consideram como base da cultura organizacional o compartilhamento de valores e crenças. Autores conhecidos como Hofstede (1980) e Schein (1993) fazem parte de tal abordagem. Numa linha mais crítica, aparece à segunda perspectiva, a da diferenciação, que reconhece as inconsistências e valoriza os conflitos surgidos a partir das subculturas naturais de uma organização. Por fim, a perspectiva da fragmentação valoriza as contradições e ambiguidades que marcam o dia a dia 
organizacional, enfatizando que são raras tanto as consistências quanto as inconsistências no ambiente das empresas.

Apesar de serem muito comuns definições da cultura organizacional como sistema de crenças de uma empresa, conceitos como o de Cavedon (2003, p. 59) são mais amplos, dando espaço para o reconhecimento das três perspectivas referidas anteriormente. Assim, segundo a autora, a cultura organizacional representa "a rede de significações que circulam dentro e fora do espaço organizacional, sendo simultaneamente ambíguas, contraditórias, complementares, díspares e análogas implicando ressemantizações que revelam a homogeneidade e a heterogeneidade organizacionais".

De qualquer modo, se reconhece que a cultura de uma organização é formada por elementos centrais, como valores, crenças e tabus que se manifestam através de diversas formas, como rituais, histórias, linguagem, práticas, ambiente de trabalho e arranjos físicos, entre outras formas. Segundo Alvesson (2002), através dessas manifestações, entende-se o simbolismo coletivo de uma organização, o que permite iluminar importantes aspectos da mesma. Nesse sentido, é importante conhecer a cultura de uma organização para entender a natureza das resistências aos processos de inovação e mudança. Para Alvesson e Sveningsson (2008), é um mito que a mudança é boa por si só. Na verdade, qualquer inovação altera a rede de relações que forma a cultura de uma organização, sistema que demora a ser reconstruído, trazendo muitas vezes descontentamentos para os membros de uma empresa que, não raras vezes, se transformam em frustração e cinismo.

Destarte pode-se afirmar que a cultura de uma organização afeta a percepção e o tipo de resistência das pessoas diante da introdução de um novo processo ou nova tecnologia. Nesse sentido, Zhu e Engels (2013), ao analisar a percepção de alunos e professores de universidades chinesas diante da introdução de inovações nas mesmas, mostram que a cultura de tais instituições afeta a percepção sobre a necessidade e o processo de implantação desses novos processos. A resistência a alguma inovação, em geral, se dá pela omissão das pessoas diante de novo processo ou através da propagação da desconfiança em relação ao mesmo, uma espécie de sabotagem viabilizada pela divulgação antecipada de um possível fracasso da mudança. Por outro lado, após a inovação completamente implantada, observa-se, com o tempo, a influência de tal alteração na cultura da organização. Desse modo, tal cultura, com o decorrer do tempo, passa de influenciadora do processo para um estado em que é modificada 
pela própria inovação. E, em geral, o funcionário que apresentou resistência, como omisso ou sabotador durante o processo, auxilia a sustentar a nova mudança.

\section{MÉTODO}

Como metodologia de pesquisa escolheu-se o estudo de caso, pois, segundo Yin (2001, p.32), “um estudo de caso é uma investigação empírica que investiga um fenômeno contemporâneo dentro de seu contexto da vida real, especialmente quando os limites entre o fenômeno e o contexto não estão claramente definidos", e, essa é a situação estudada. Além disso, o método escolhido torna-se pertinente por ser muito difícil separar o contexto do controle e manuseio de documentos de uma IES e o reflexo do mesmo sobre gestão da IES, devido a seu dinamismo e possibilidade de influenciar constantemente.

Dentre os tipos classificados por Yin (2001) o que mais se encaixa neste trabalho é o estudo de caso explanatório. Pretende-se que os eventos analisados possam traduzir-se em explanações utilizáveis, servindo como base em outras situações.

\subsection{COLETA DE DADOS}

Como processo de coleta de dados acompanhou-se a implantação de nova sistemática baseada em tecnologia eletrônica GED. Objetivou-se verificar o processo sob os pontos de vistas tecnológicos e pessoais, iniciado em 01/01/2011 e finalizado em 01/01/2013.

$\mathrm{Na}$ abordagem tecnológica foram analisados documentos do processo anterior (microfilmagem) executado de forma manual e comparados com os posteriores à implantação, para que se fosse possível avaliar questões de acuracidade, esforço, agilidade na execução, necessidade de mão de obra, entre outras.

Além disso, para se entender os aspectos pessoais foram executadas entrevistas em profundidade apoiadas por roteiro semiestruturado com o gestor, que atua a 5 anos nesse setor com formação na área de gestão e Pós-Graduação Stricto Sensu. Também, os funcionários responsáveis pela digitalização dos documentos (15 e 10 anos no setor) foram entrevistados objetivando enriquecer o estudo.

\subsection{ANÁLISE DE DADOS}

Para análise de dados utilizou-se análise de conteúdo categorizando-se as dimensões (BARDIN, 2009) de acuracidade, agilidade, custo e mão de obra. Entendeu-se que estas 
categorias representaram os principais aspectos envolvidos permitindo analisar as questões técnicas e pessoais relacionado-as com a cultura da instituição.

\subsection{IES OBJETO DE ESTUDO}

O objeto de estudo foi a Universidade de Caxias do Sul, no Rio Grande do Sul. Esta Universidade, comunitária e regional foi constituída em 1967 a partir do interesse da sociedade civil e do poder público estando presente, além de Caxias do Sul, onde possui sua sede, nos municípios de Bento Gonçalves, Vacaria, São Sebastião do Caí, Canela, Nova Prata, Guaporé, Farroupilha e Veranópolis contando com atualmente mais de 37.000 alunos na graduação, pós-graduação, extensão, ensino médio e técnico em mais de 160 ofertas de cursos (UNIVERSIDADE DE CAXIAS DO SUL, 1992; UCS, 2013).

Seu caráter comunitário e regional mantém, além dos cursos de graduação e pósgraduação, programas permanentes de extensão, projetos de pesquisa em conjunto com o poder público e sua presença propicia uma "capacidade de articulação das diferentes forças econômicas, sociais e políticas de cada localidade e da região como um todo" (ZORZI, 1992).

\section{COMPARATIVO ENTRE OS PROCESSOS}

A IES estudada sempre manuseou os documentos com esmero e cuidado, mas dentro de um paradigma físico. Portanto, cópias xerográficas e grandes cofres de armazenamento para os arquivos foram objetos comuns de uso. Pode-se dizer que a segurança necessitava e podia ser vista a olhos nus. Este processo era intenso em mão de obra, ocupação de espaço físico e tempo para execução de todas as tarefas. Além disso, devido ao contínuo manuseio humano passível de erros que afetavam diretamente ao cliente podendo, inclusive, acarretar processos jurídicos com substanciais perdas financeiras.

$\mathrm{Na}$ verdade a IES já enfrentou situações, tais como: Distância entre as unidades universitárias, extravio e descarte indevido de documentos de alunos (certidões, históricos, comprovante de ensino médio, identificação, conteúdos programáticos, autorizações etc.), duplicações de documentos.

Em seu processo de microfilmagem, apenas o aluno concluinte de um curso ou que perdia o vínculo acadêmico tinha guarda perpétua permanecendo os demais documentos espalhados em arquivos específicos em cada uma das oito unidades da IES. Ainda assim, para ter acesso aos documentos às unidades solicitavam formalmente a busca ao setor responsável 
que ao localizar o documento providenciava cópia que era enviada via fax ou sistema de correio interno para o solicitante. Nesse último processo, o tempo médio de disponibilização do documento era de 36 horas após a solicitação.

Assim, a conversão do modelo de microfilmagem, centralizado na sede da IES (preparo, produção e consulta) para o modelo de digitalização com aplicação da certificação digital padrão ICP Brasil, além de qualificar e tornar perpétua a guarda dos documentos, através da intranet, torna acessível o acesso aos documentos acadêmicos através do sistema Ábaris (software de indexação e busca dos documentos) socializa a informação e qualifica o atendimento ao usuário conforme demonstrado na Figura 1.

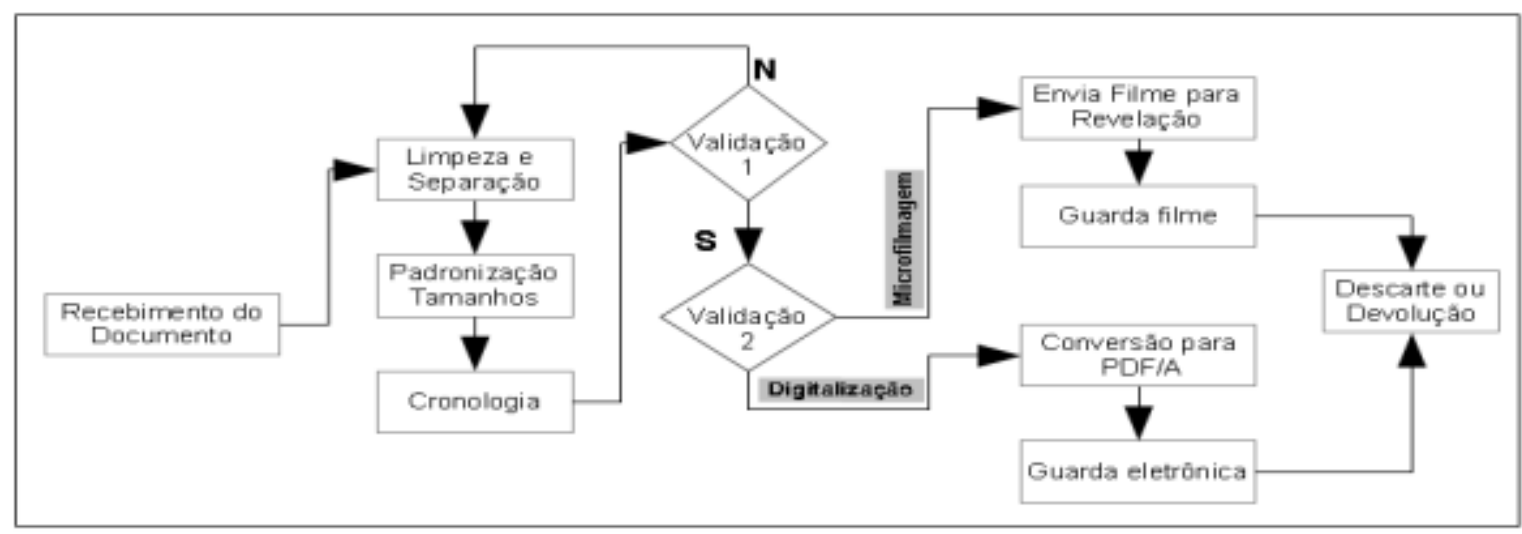

Figura 1 Fluxograma do processo de guarda na IES Fonte: Elaborado pelos autores.

Nesse novo método (digitalização) todo o processo de preparo dos documentos para a captura da imagem é similar ao método da microfilmagem. Dentre as qualidades percebidas, além da agilidade, o tratamento da imagem (clareamento ou escurecimento) e a indexação ao cadastro único do aluno na IES são realizados automaticamente pelo software de captura, indexação e transformação em PDF/A das imagens (Kofax) através de padronização de campos específicos de captura de dados. Já documentos históricos da IES após a digitalização das imagens estes retornam ao arquivo do centro de documentação da IES para guarda adequada.

\section{DISCUSSÕES SOBRE OS RESULTADOS E CONSIDERAÇÕES FINAIS}

Os antigos sistemas de arquivamento ou guarda de documentos nas organizações e, neste caso, nas universidades, tem se tornando um grande problema por questões de espaço 
físico e preservação da integridade dos documentos acadêmicos. Nas IES a guarda destes documentos é caracterizada, na sua maioria, como guarda perpétua.

Porém no ambiente acadêmico, estes documentos devem estar a todo o momento disponível e de fácil acesso e, a digitalização é uma destas formas, de se ter acesso rápido, eficiente para o atendimento. Por ser mais econômica, tanto de tempo quanto de custos, a digitalização com certificação digital garante a legitimidade do documento tornando o meio eletrônico auxiliar neste processo (HASSLER e BIELY, 1999; WILSON, 1999). Além disso, o processo de digitalização com certificação digital é uma solução legal e capaz de estancar ou diminuir a geração de documentos em papel pelas Secretarias Acadêmicas e melhorando as condições de preservação (WATERS, 2001; CONWAY, 2001).

Ressalta-se também que tal tecnologia de arquivamento e guarda, mesmo que referendada pela Lei 12.265 , não possui uniformidade de entendimento das temporalidades nem, tampouco, da evolução dos sistemas computacionais e de guarda e gerenciamento de informações, mesmo após o fenômeno Big Data.

Um efeito que pode ser observado no processo renovado foi à quebra do paradigma do documento físico, no qual a ideia de quantidade e segurança podia ser vista a olhos nus através de grandes arquivos metálicos com potentes trancas externas, com ocupação de grande área construída (prédios, depósitos) e, por vezes em locais inapropriados sem o devido zelo. Outro aspecto apontando pelos entrevistados foi a integridade dos dados que não puderam mais ser alterados depois de gerados (MARQUES, 2005), por exemplo, uma troca de nota por parte de um professor somente pode ser procedida se houver um cumprimento de uma protocolo interno, anteriormente era facilitado devido a um controle centralizado menor e a facilidade de acesso ao documento.

Observou-se, pelas entrevistas que, embora num primeiro momento a insegurança devido à visualização e manuseio físico dos arquivos se manifestasse, na medida em que o tempo transcorria e as consultas se repetiam com precisão esta situação tendeu a desaparecer modificando a percepção dos atores baseada na lógica anterior (ZHU e ENGELS, 2013). Este fato foi reforçado pelo gestor da área ao alegar que as pessoas ao sentirem-se a vontade no uso da nova tecnologia tornaram-se mais eficazes nas respostas das pesquisas documentais, visto que cada aluno, independente da unidade ao qual pertence seu curso (a IES pesquisada possui unidades fora da sede) passou a possuir apenas um local de guarda e recuperação de seus documentos promovendo a acuracidade (PEREIRA, 2012). 
O gestor argumentou, também, que a resistência para a mudança de tecnologia foi mais evidente nas unidades fora da sede da IES que não estavam amplamente preparadas para a perda dos arquivos físicos com as pastas dos documentos dos alunos de sua unidade acadêmica. Ainda assim, como existiam arquivos com pastas de alunos em todas as unidades acadêmicas durante a migração houve momentos em que o tempo entre o recebimento dos documentos, seu preparo, digitalização e disponibilização para o usuário coincidiu com a necessidade de manuseio, tendo o usuário que aguardar cerca de 5 dias para visualizar o documento digitalizado.

Atualmente, após três anos de implantação, mais de 30.000 .000 (trinta milhões) de processos estão digitalizados, certificados digitalmente e disponíveis para os usuários da IES (secretaria acadêmica, coordenadores de curso). Além dos documentos acadêmicos, projetos de pesquisa, projetos de cursos de pós-graduação, fotos, dissertações e teses (com restrição de acesso) foram incluídos no projeto e, segundo o gestor tornou-se uma ferramenta fundamental de auxílio para a gestão organizacional.

Sob o ponto de vista dos funcionários entrevistados, a nova tecnologia permitiu que documentos até então guardados em outros locais (gavetas, armários e outros arquivos setoriais) gradativamente migraram para uma base única, transformando a guarda pessoal de documentos para institucional. Do ponto de vista organizacional (ORTT e DUIN, 2008), o gestor e os funcionários concordaram mencionando que: esta flexibilização refletiu em melhora nos tempos de resposta na recuperação dos documentos, na redução do esforço físico, na unicidade dos documentos (GAVILANES, 2005), ou seja, a IES está aprendendo (NONAKA e TAKEUSHI, 1997; SENGE, 1999) como tratar seus documentos em geral, nos mais diversos setores.

Além disso, um aspecto importante foi de a digitalização ter sido implementada em um setor crítico devido à especificidade da guarda perpétua de documentos, mas em virtude do êxito está sendo implantada em outros setores da IES melhorando a receptividade da cultura dos usuários a respeito da inovação. Abrindo a possibilidade para uma nova cultura (HOFSTEDE, 1980; SCHEIN, 1993; MARTIN, 2002) com a utilização de ferramentas mais atualizadas como a tecnologia Big Data. 


\subsection{SUGESTÕES PARA A IES}

As Instituições de Ensino Superior, ao aplicarem as tecnologias de digitalização com certificação digital, poderão trocar, com segurança (HASSLER e BIELY, 1999; WILSON, 1999), arquivos de alunos em procedimento de transferências, solicitação de documentos de aproveitamento de estudos entre outros. Ainda assim, internamente, o lançamento dos registros de notas de alunos, solicitações protocolares, autorizações eletrônicas de processos entre outros procedimentos poderiam ser incluídos com segurança e baixo investimento pela aplicação da assinatura pelo certificado digital, tornando possível, além da disponibilização imediata dos documentos e sua guarda, a inclusão de procedimentos de inteligência do uso de seu conteúdo.

No caso da IES estudada, como se caracteriza pela inovatividade no processo de guarda e manuseio de documentos, sugere-se que se utilize da tecnologia "big data" onde capturaria além dos atuais documentos do sistema de guarda digitalizado outras informações provenientes de outras fontes: telefone; redes sociais; mídias tradicionais, e-mails, etc. Com isto ampliaria fortemente sua capacidade de gerar estratégias mais assertivas na captação de necessidades e atendimento de seu publico alvo.

\section{REFERÊNCIAS}

ALVESSON, M.; Understanding organizational culture. London: Sage, 2002.

ALVESSON, M.; SVENINGSSON, S.; Changing organizational culture: cultural change work in progress. London: Routledge, 2008.

AUSTER, E.; CHOO, C. W. CEOs, information, and decisionmaking: scanning the environment for strategic advantage. Library Trends, v. 43, n. 2, p. 206-225, Fall 1994.

BARDIN, L. Análise de Conteúdo. Lisboa, Portugal; Edições 70, LDA, 2009.

BIG DATA: The next frontier for innovation, competition, and productivity. McKinsey Global Institute, 2011.

BRASIL. Instituto Nacional de Tecnologia da Informação. Disponível em: $<$ http://www.iti.gov.br/twiki/bin/view/Certicacao/WebHome>. Acesso: 9 jul. de 2012a.

.LEI No 5.433. Disponível em: <www.planalto.gov.br/ccivil_03/leis/L5433.htm>.7 Acesso em: 28 jun. 2012 b. 
.LEI No 10.973-2004. Disponível em:

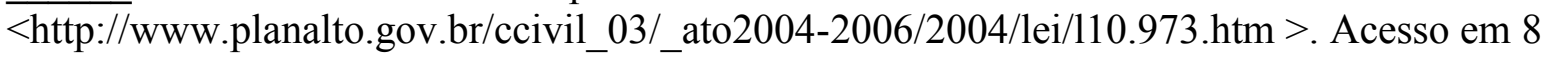
jul. de 2012c.

LEI No 4.915-2003. Disponível em:

$\overline{<\mathrm{http}: / / w w w . s i g a . a r q u i v o n a c i o n a l . g o v . b r / c g i / c g i l u a . e x e / s y s / s t a r t . h t m>. ~ A c e s s o ~ e m ~} 2$ fev. de $2013 \mathrm{e}$.

.Portaria no 1.224-2013. Disponível em:

$<$ http://www.siga.arquivonacional.gov.br/cgi/cgilua.exe/sys/start.htm?infoid=288\&sid=170\&t $\mathrm{pl}=$ printerview $>$ Acesso em 02 fev. de $2013 \mathrm{f}$

.Medida Provisória n..$^{\circ}$ 2.200. Disponível em:

<http://www.iti.gov.br/twiki/bin/view/Certificacao/MedidaProvisoria $>$. Acesso em: 27 jun. de $2012 \mathrm{f}$.

BURNETT, S.; PAINE, S. Criptografia e Segurança - O Guia Oficial da RSA. Editora Campus, 2002.

CAVEDON, N. R. Antropologia para administradores. Porto Alegre: Editora da URGS, 2003.

CERTSIGN Certificadora Digital S.A. Disponível em: $<$ http://www.certisign.com.br/ $>$. Acesso em: 25 mar 2014.

CHANAL, V. Innovation management and organizational learning: a discursive approach. European Journal of Innovation Management, v. 7, n. 1, p. 56-54, 2004.

CONWAY, P. Preservação no universo digital. 2.ed. Rio de Janeiro: Arquivo Nacional, 2001.

CUMMING. B. S. Innovation overview and future challenges. Journal of Innovation Management, v. 1, n. 1, p. 21-29, 1998.

CONARQ. Legislação Arquivistica Brasileira, Conselho Nacional de Arquivos - versão digital para download de maio de 2012. Disponível em:

$<$ http://www.conarq.arquivonacional.gov.br/cgi/cgilua.exe/sys/start.htm?sid=48. $>$ Acesso em 27 jun. 2012.

DAVENPORT, T, PRUSAK, L. Conhecimento Empresarial. Rio de Janeiro: Campus, 1998.

DEAN, J. W. Building the Future: The justification process for new technology. In:

Pennings, J.M., Buitendam, New Technology as Organizational Innovation. Ballinger: Cambridge, 1987. 
FREEMAN, E. Digital Signatures and Electronic Contracts, EDPACS: The EDP Audit, Control \& Security Newsletter, v. 32, n. 3, p. 18-24, 2004.

GAVILANES, J. C. D. A certificação digital como instrumento de garantia da segurança e credibilidade da informação digital: um estudo de caso brasileiro. Faculdade IBMEC Programa de Pós-Graduação e Pesquisa em Administração e Economia. Rio de Janeiro: 2005.

HART, S. New Product Development. London: Dryden Press, 1996.

HASSLER, V. BIELY, H. Digital signature management. Eletronic Networking Applications and Policy, v. 9, n. 4, p. 262-271, 1999.

HOFSTEDE, G.; Culture's consequences: international differences in work related values. Beverly Hills: Sage, 1980.

JOHNE, A. Successful market innovation. European Journal of Innovation Management, v. 2, n. 1, p. 6-11, 1999.

KUHN, R. L. Frontiers in creative and innovative management. Ballinger: Cambridge, 1985.

LAUDON, K.C.; LAUDON, J. P. Sistemas de informação gerenciais: administração a empresa digital. São Paulo: Prentice Hall, 2004.

LINCOLN, A. Electronic Signature Laws and the Need for Uniformity in the Global Market. Journal Small \& Emerging Bus, p. 67-86, 2004.

MARQUIS, D. G. The anatomy of successful innovations. Innovation, November, 1969.

MARTIN. J.; Organizational culture: mapping the terrain. Thousand Oaks, CA: Sage, 2002.

MARTIN, J.; FROST, P.; Jogos de guerra da cultura organizacional: a luta pelo domínio intelectual. In: CLEGG, Stewart; HARDY, Cynthia; NORD, Walter R. Handbook de estudos organizacionais: modelos de análise e novas questões em estudos organizacionais. São Paulo: Atlas, vol. 2, p. 219-251, 2001.

MEC, Ministério da Educação. Portaria n. ${ }^{\circ}$ 40. Disponível em:

$<$ www.portal.mec.gov.br/seed/arquivos/pdf/ead/port_40.pdf $>$. Acesso em: 8 jun. 2012.

.Portaria SeSu 255 Portaria n²55, de 20 de dezembro de 1990. Disponível em:

<www.ufrgs.br/cepe/legislacao/Portaria\%20255-90\%20MEC.pdf.> Acesso em 16 jun. 2012

NONAKA, I.; TAKEUCHI, H. Criação do Conhecimento na Empresa. Rio de Janeiro: Campus, 1997.

ORTT, J. R.; DUIN, P. A.V.D. The evolution of innovation management towards contextual innovation. European Journal of Innovation management, v. 11, n. 4, p. 522-538, 2008.

PEREIRA, C. B. Entenda a validação jurídica dos arquivos eletrônicos. Disponível em: $<$ http://www.cleber.com.br/certifica2.html $>$. Acesso em: 10 jul. 2012

PETTIGREW, A. On studying organizational cultures. Administrative Science Quarterly, v. 24, n. 4, p. 570-581, 1979.

PORTER, M. Competitive Strategy: Techniques for Analyzing Industries and Competitors. New York: The Free Press, 1980.

SCHEIN, Edgar H. Organizational culture and leadership. São Francisco, CA: JosseyBass, 1992. 
SENGE, P. M. A Quinta Disciplina: Arte, Teoria e Prática da Organização de Aprendizagem. São Paulo: Best Seller, 1999.

SMEDINGHOFF, T. J.; BRO, R. H. Moving With Change: Electronic Signature Legislation As A Vehicle For Advancing E-Commerce. The John Marshall Journal of Computer \& Information, v. 17, n. 3, 1999.

SMIRCICH, L. Concepts of culture and organizacional analysis. Administrative Science Quaterly, Ithaca, v. 28, n. 3, p. 339-358, 1983.

MARQUES, A. T. G. L.; A prova documental na internet: validade e eficácia do documento eletrônico. Curitiba: Juruá, 2005.

THE ECONOMIST, A Special Report on Managing Information: Data, Data

Everywhere. Disponível em: $<$ http://www.economist.com/node/15557443> Acesso em 05 mar. 2014.

UCS - Universidade de Caxias do Sul. Disponível em: http://www.ucs.br/site/institucional/, Acesso em 12 fev. 2013.

UDWADIA. F. E. Creativity and innovation in organizations. Technological Forecasting and Social Change, v. 38, n. 1, p. 66, 1990.

UNCITRAL. United Nations Commission on International Trade Law. Disponível em: $<$ www.uncitral.org/pdf/english/workinggroups/wg_ec/wp-71.pdf $>$ acesso em 09 jul. 2012

UNIVERSIDADE DE CAXIAS DO SUL. A regionalização da Universidade: conceitos e perspectivas. Caxias do Sul, RS: UCS, [1992].

WATERS, D. J. Do microfilme à imagem digital: como executar um projeto para estudo dos meios, custos e benefícios de conversão para imagens digitais de grandes quantidades de documentos preservados em microfilme. 2.ed. Rio de Janeiro: Arquivo Nacional, 2001.

WILSON, S. Digital signatures and the future of documentation. Information Management \& Computer Security, p. 83-87, 1999.

WINN, J. K. The Emperor's New Clothes: The Shocking Truth About Digital Signatures and Internet Commerce, 37 Idaho L. Rev. 353, 2001.

ZHU, C.; ENGELS, N. Organizational culture and instructional innovations in higher education: Perceptions and reactions of teachers and students. Educational Management Administration \& Leadership, v. 42, n. 1, p. 136-158, 2013.

ZORZI, I. Universidade de Caxias do Sul. A regionalização da Universidade: conceitos e perspectivas. Caxias do Sul, RS: UCS, 1992. 